Recepción: 15 / 04 / 2017

Ciencias Económicas

Aceptación: 01 / 05 / 2017

Articulo Científico

Publicación: 15 / 05 / 2017

\title{
Propuesta de comercialización de productos de cuero vacuno al mercado Europeo
}

\author{
Proposal for the marketing of beef products on the \\ European market
}

\section{Proposta comercialização de produtos para o couro do mercado Europeu}

Sidia E. Vera-Gutiérrez sidiavera@yahoo.com

Janet P. Pantoja-Rodríguez" janet.pantojaro@ug.edu.ec

Teresa Y. Avilés-Flor "' avilesft@ug.edu.ec

Correspondencia: sidiavera@yahoo.com

Magister Ejecutivo en Dirección de Empresas con Énfasis en Gerencia Estratégica; Diplomado Superior en Gerencia de Marketing; Especialista en Gerencia de Proyectos; Ingeniera Comercial; Contadora Publica Autorizada; Universidad Agraria del Ecuador, Ecuador.

I. $\quad$ Master en Dirección Comercial y Marketing; Diploma Superior en Docencia Universitaria; Ingeniero Comercial; Profesora Especialización Educación Primaria; Universidad de Guayaquil, Ecuador.

III. Magister Ejecutiva en Dirección de Empresas con Énfasis en Gerencia Estratégica; Especialista en Gerencia de Proyectos; Diplomado Superior en Gerencia de Marketing; Ingeniera Comercial; Contadora Publica Autorizada; Universidad de Guayaquil, Ecuador. 


\section{Resumen}

El presente estudio se enfocó al proceso de internacionalización de la marca Legere, de accesorios hechos a mano de cuero, al mercado español. La investigación se basó en cifras estadísticas de comercio exterior, tendencias de consumo, encuestas a los potenciales clientes y entrevistas. Los productos tienen gran aceptación por su calidad y diseño. El trabajo está divido en seis apartados. En el primer capítulo, se introduce el tema estudiado, su coyuntura así como los antecedentes que motivaron su investigación. Se hace hincapié en su importancia práctica y teórica. A continuación, se aporta con un marco teórico que contextualiza al lector en el enfoque planteado. Se recabó información cualitativa de fuentes primarias y oficiales para crear un perfil general de los artículos de cuero y de comercio exterior entre ecuador y España. En el tercer apartado, se detallan los métodos y herramientas empleados para llevar a cabo la investigación y se describe el proceso para conducir las encuestas y entrevistas y sus resultados principales. En el siguiente capítulo, se construyó la propuesta de internacionalización a partir de la investigación. Está conformada por los siguientes ejes básicos: mercados, promoción, ubicación y presupuestos. La discusión de los resultados y las conclusiones y recomendaciones conforman el quinto y el apartado final del trabajo.

Palabras clave: Internacionalización; mercado español; comercio exterior. 


\begin{abstract}
The present study focused on the process of internationalization of the Legere brand of handmade leather accessories to the Spanish market. The research was based on statistics of foreign trade, consumer trends, surveys of potential clients and interviews with experts. The products have great acceptance for their quality and design. The work is divided into six sections. In the first chapter, it introduces the subject studied, its conjuncture as well as the antecedents that motivated its investigation. Emphasis is placed on its practical and theoretical import. Next, it is provided with a theoretical framework that contextualizes the reader in the approach proposed. Qualitative information was collected from primary and official sources to create a general profile of leather goods and foreign trade between Ecuador and Spain. The third section details the methods and tools used to conduct the research and describes the process for conducting surveys and interviews and their main results. In the next chapter, the proposal of internationalization was built from the research. It is made up of the following basic axes: markets, promotion, location and budgets. The discussion of the results and conclusions and recommendations form the fifth and final section of the paper.
\end{abstract}

Key words: Internationalization; spanish market; foreign trade. 


\section{Resumo}

O presente estudo incidiu sobre o processo de internacionalização da marca Legere de acessórios de couro artesanal para o mercado espanhol. A pesquisa foi baseada em estatísticas de comércio exterior, tendências de consumo, pesquisas de clientes potenciais e entrevistas com especialistas. Os produtos têm grande aceitação para a sua qualidade e design. $O$ trabalho é dividido em seis seções. No primeiro capítulo, introduz o sujeito estudado, sua conjuntura e os antecedentes que motivaram sua investigação. A ênfase é colocada em sua importância prática e teórica. Em seguida, é fornecido um quadro teórico que contextualiza o leitor na abordagem proposta. Foram coletadas informações qualitativas de fontes primárias e oficiais para criar um perfil geral de couro e comércio exterior entre o Equador ea Espanha. A terceira seção detalha os métodos e ferramentas usadas para conduzir a pesquisa e descreve o processo para conduzir pesquisas e entrevistas e seus principais resultados. No próximo capítulo, a proposta de internacionalização foi construída a partir da pesquisa. É constituído pelos seguintes eixos básicos: mercados, promoção, localização e orçamentos. A discussão dos resultados e conclusões e recomendações formam a quinta e última seção do artigo.

Palavras chave: Internacionalização; mercado espanhol; Comércio exterior. 


\section{Introducción.}

Legere es un emprendimiento joven ecuatoriano que fabrica artículos en cuero para complementar el vestuario masculino. Son accesorios hechos a mano, con alta calidad, diseños exclusivos, variedad de colores y texturas. El cuero es una de las materias primas más nobles y de mayor durabilidad en el mercado, y este emprendimiento lo utiliza transformándolo con cada detalle en su proceso productivo, alcanzando un alto nivel de excelencia y elegancia. (Águeda \& Mondéjar, 2013)

La empresa posee dos líneas de productos, una en cuero y otra textil. La primera incluye una gama de accesorios de uso cotidiano, como porta celulares, billeteras, porta tarjetas, porta chequeras, monederos, porta pasaportes, entre otros. En la línea textil, Legere tiene accesorios para la vestimenta del hombre, como pañuelos, broches, corbatas y corbatines. Cada producto está diseñado y producido bajo altos estándares de innovación, lo cual permite que Legere alcance la escala máxima de diferenciación en el mercado, formando en los clientes un estilo de vida asociado a la marca, en la cual se denota sutileza y practicidad (Legere, 2015).

El producto es realizado por artesanos ecuatorianos localizados en la ciudad de Guayaquil, la materia prima es $100 \%$ cuero vacuno de nivel AAA. Los artesanos dedicados a esta actividad son estrictamente seleccionados, brindando especial cuidado a la calidad de los productos. En el Ecuador, este negocio maneja una comercialización a través de la distribución autorizada en varios puntos del país, como: Guayaquil, Quito, Portoviejo y Cuenca. (Arciniega, 2015)

Además la marca cuenta con certificaciones locales de ser un producto ecuatoriano con excelencia. Todos estos elementos permiten que el producto tenga grandes posibilidades de aceptación en el mercado exterior. 
Ecuador es uno de los países de mayor diversidad, la producción artesanal se efectúa a gran escala, tanto así que han creado a lo largo de los años una fuerte presencia en el mercado local. Ese alto nivel obtenido ha sido el motor para que los empresarios ecuatorianos se interesen por comercializar sus productos en el exterior. La ventaja de esta comercialización es que no solo se exporta el producto sino también la imaginación y creatividad de los artesanos ecuatorianos, quienes con la habilidad de sus manos laboriosas plasman hermosas obras de arte en cada artículo. (Avila, 2011) (Braid, 2012)

La presente investigación se realiza con el objetivo de desarrollar una propuesta que permita a partir del 2017 la comercialización de los productos de cuero de la empresa Legere en la Unión Europea, para mejorar el volumen de ventas actual.

\section{Materiales y métodos.}

Se conducirá una investigación explicativa dado que se desea revelar aspectos de los componentes del fenómeno a investigar con apoyo en revisiones documentales. La investigación documental mediante la cual se obtuvo información de textos, documentos, proyectos, revistas e internet para recopilar la información necesaria en esta investigación, así como los datos verídicos provenientes de las diferentes instituciones del estado ecuatoriano y del mercado europeo.

Simultáneamente, se realizará una investigación cuantitativa al analizar las estadísticas poblacionales del mercado potencial europeo para la comercialización de los productos de cuero, así como de comercio exterior. Estos esfuerzos se complementarán con encuestas en el mercado español, pues el estado del arte confirmó que el mercado español es el ideal para los objetivos del proyecto. Igualmente, se emplearán métodos cualitativos al analizar entrevistas con expertos que proveerán sus experticias y perspectivas sobre la temática abordada. 
Métodos

El método con el cual se desarrollará la investigación es el deductivo, pues se van a tomar ciertos aspectos generales para llegar a conclusiones específicas que apoyen las motivaciones del presente estudio.

\section{Estadística descriptiva}

La estadística se recogerá de fuentes oficiales y entidades públicas, como el Banco Central del Ecuador y organismos de la Unión Europea.

\section{Población y muestra}

El plan de comercialización de Legere hacia España se dirige al mercado de Barcelona, provincia española situada en el nordeste del país. Su capital es Barcelona, donde viven casi el 30\% de los 5,529.099 habitantes de la provincia. 


\section{Resultados.}

\section{¿Cuáles de los siguientes artículos compra con mayor frecuencia?}

\begin{tabular}{|c|c|c|}
\hline \multicolumn{3}{|c|}{ TABLA DE FRECUENCIA } \\
\hline DETALLE & DATOS & PORCENTAJE \\
\hline Bolso & 169 & $44.20 \%$ \\
\hline Monedero & 75 & $19.62 \%$ \\
\hline Billetera & 58 & $15.14 \%$ \\
\hline Porta Laptop & 23 & $6.06 \%$ \\
\hline Llavero & 12 & $3.03 \%$ \\
\hline Porta tarjeta & 12 & $3.03 \%$ \\
\hline Maletón & 12 & $3.03 \%$ \\
\hline Porta pasaporte & 12 & $3.03 \%$ \\
\hline Porta chequera & 6 & $1.57 \%$ \\
\hline Porta llave & 5 & $1.31 \%$ \\
\hline TOTAL & $\mathbf{3 8 2}$ & $\mathbf{1 0 0 \%}$ \\
\hline
\end{tabular}

\section{Tabla $N^{\circ}$ 1.- Artículos comprados con mayor frecuencia}

De los diez productos más comunes fabricados en cuero, el adquirido con mayor frecuencia es el bolso. No es de extrañar pues, a simple vista, los bolsos de cuero son las más utilizados en el día día, sobre todo como complemento del uniforme de oficina. Al ser un implemento de uso diario, seria el más opcionado por el público por su resistencia y durabilidad. Dos quintas partes de los encuestados se decantaron por este producto.

En segundo lugar, ostentando casi una quinta parte de la muestra cada uno, están el monedero y la billetera. Son dos de las prendas más utilizadas tanto por hombres y mujeres. En tercer lugar, con menos de una décima parte de los interrogados, cada uno, están los portalaptop, llaveros, portatarjeta, maletín, portapasaporte, portachequera y portallavero. Esto podría explicarse porque en el mercado existen diversos materiales para este tipo de acccesorios. Por ejemplo, para los portalaptop hay variedades en neopreno, microfibra o lona. 
De manera general, ¿cada cuánto tiempo usted compra este tipo de productos?

\begin{tabular}{|c|c|c|}
\hline \multicolumn{3}{|c|}{ TABLA DE FRECUENCIA } \\
\hline DETALLE & DATOS & PORCENTAJE \\
\hline Anual & 197 & $51.52 \%$ \\
\hline Trimestral & 93 & $24.24 \%$ \\
\hline Mensual & 46 & $12.12 \%$ \\
\hline Semestral & 46 & $12.12 \%$ \\
\hline TOTAL & $\mathbf{3 8 2}$ & $\mathbf{1 0 0 \%}$ \\
\hline
\end{tabular}

\section{Tabla $N^{\circ}$ 2.- Tiempo en el que adquiere productos de cuero}

El objetivo de esta pregunta fue conocer la frecuencia con la cual los consumidores adquieren un producto de cuero. De acuerdo a los resultados, la mitad de los encuestados afirman que compran un accesorio de cuero una vez al año. Casi la cuarta parte de la muestra contestó que compra productos de cuero cuatros veces al año o cada tres meses. Finalmente, un poco más de la décima parte afirmó que obtiene este tipo de accesorios cada mes o dos veces al año, respectivamente.

¿Cuáles son los motivos de compra de artículos de cuero? Ordene del 1 al 4 siendo 1 el más importante.

\begin{tabular}{|c|c|c|}
\hline \multicolumn{3}{|c|}{ TABLA DE FRECUENCIA } \\
\hline DETALLE & DATOS & PORCENTAJE \\
\hline Uso personal & 150 & $39.39 \%$ \\
\hline Obsequio para un familiar & 139 & $36.36 \%$ \\
\hline Obsequio para amigos & 58 & $15.15 \%$ \\
\hline Obsequio corporativo & 35 & $9.09 \%$ \\
\hline TOTAL & $\mathbf{3 8 2}$ & $\mathbf{1 0 0 \%}$ \\
\hline
\end{tabular}

Tabla $N^{\circ}$ 3.- Motivo por el que compran los productos de cuero 
Con esta interrogante, se conocieron las razones tras la compra de un artículo de cuero. El $39 \%$ de los encuestados señalaron que realizan la adquisición para uso personal. En similar proporción, los interrogados respondieron que la compra corresponde al obsequio para un familiar. En tercer lugar, menos de una quinta parte de la muestra contestó que los artículos son destinados para un amigo. Finalmente, un poco menos de la décima parte del grupo indicó que la adquisición tiene fines corporativos.

\section{Indique del siguiente listado cuales son los criterios que usted considera más importantes para} la compra de artículos de cuero. Siendo 1 el más importante y 5 el menos.

\begin{tabular}{|c|c|c|}
\hline \multicolumn{3}{|c|}{ TABLA DE FRECUENCIA } \\
\hline DETALLE & DATOS & PORCENTAJE \\
\hline Precio & 3.7 & $24.50 \%$ \\
\hline Elegancia y status & 3.4 & $22.52 \%$ \\
\hline Calidad & 2.9 & $19.21 \%$ \\
\hline Diseño y moda & 2.9 & $19.21 \%$ \\
\hline Facilidad de uso & 2.2 & $14.57 \%$ \\
\hline TOTAL & $\mathbf{1 5}$ & $\mathbf{1 0 0 \%}$ \\
\hline
\end{tabular}

Tabla $N^{\circ}$ 4.- Aspectos atractivos del producto

En esta pregunta se han escogido cinco categorías que ayudan a definir qué aspecto más atrae al cliente de los productos de cuero. Definitivamente, la facilidad de uso está en primer lugar. Le sigue la importancia en la calidad y diseño de moda, ambos cada uno con una calificación promedio de 2.9. En último lugar está el precio, por tanto se entiende que a este mercado no le importaría pagar un poco más a cambio de un producto de buena calidad. Estas respuestas son muy importantes para delinear la estrategias de marketing del producto. 
En el caso específico de la compra de una billetera para caballero, ¿cuánto es su presupuesto promedio?

\begin{tabular}{|c|c|c|}
\hline \multicolumn{3}{|c|}{ TABLA DE FRECUENCIA } \\
\hline DETALLE & DATOS & PORCENTAJE \\
\hline$€ 20-€ 40$ & 197 & $51.52 \%$ \\
\hline$€ 41-€ 60$ & 162 & $42.42 \%$ \\
\hline$€ 61-€ 80$ & 23 & $6.06 \%$ \\
\hline$€ 81$ y más & 0 & $0.00 \%$ \\
\hline TOTAL & $\mathbf{3 8 2}$ & $\mathbf{1 0 0 \%}$ \\
\hline
\end{tabular}

Tabla $N^{\circ}$ 5.- Presupuesto para adquirir una billetera de cuero

El precio de los productos es una característica muy importante a considerar en el sector comercial. Por una billetera de cuero, la mitad de los encuestados señalaron que estarían dispuestos a pagar un valor entre $€ 20$ a $€ 40$. Dos quintas partes de los interrogados, en cambio, indicaron que les gustaría cancelar un precio en el intervalo de $€ 41$ a $€ 60$. Solo menos de la décima parte de los interrogados señaló que podría pagar hasta $€ 80$.

\section{¿Dónde adquiere sus productos de cuero?}

\begin{tabular}{|c|c|c|}
\hline \multicolumn{3}{|c|}{ TABLA DE FRECUENCIA } \\
\hline DETALLE & DATOS & PORCENTAJE \\
\hline Centros comerciales, tiendas & 232 & $60.61 \%$ \\
\hline Mercadillos artesanales & 81 & $21.21 \%$ \\
\hline Compras online & 58 & $15.15 \%$ \\
\hline Boutiques exclusivas & 12 & $3.03 \%$ \\
\hline TOTAL & $\mathbf{3 8 2}$ & $\mathbf{1 0 0 \%}$ \\
\hline
\end{tabular}

\section{Tabla $N^{\circ}$ 6.- Lugares opcionados para la compra de productos de cuero}

Entre los sitios más opcionados para adquirir los productos de cuero se encuentran los centros comerciales y tiendas, ostentando alrededor de tres quintas partes de la muestra. En segundo lugar, con una quinta parte de los entrevistados, están los mercaditos artesanales. El 15\% de los 
encuestados prefieren las compras online. En tanto que las boutiques exclusivas (3\%) no gozan de la misma acogida que las primeras opciones.

\section{¿Considera que el mercado de artículos de cuero en su ciudad ya se encuentra saturado?}

\begin{tabular}{|c|c|c|}
\hline \multicolumn{3}{|c|}{ TABLA DE FRECUENCIA } \\
\hline DETALLE & DATOS & PORCENTAJE \\
\hline Indecisos & 197 & $51.52 \%$ \\
\hline En desacuerdo & 127 & $33.33 \%$ \\
\hline De acuerdo & 35 & $9.09 \%$ \\
\hline Muy en desacuerdo & 12 & $3.03 \%$ \\
\hline Muy de acuerdo & 12 & $3.03 \%$ \\
\hline TOTAL & $\mathbf{3 8 2}$ & $\mathbf{1 0 0 \%}$ \\
\hline
\end{tabular}

\section{Tabla $N^{\circ}$ 7.- Saturación del mercado de cuero}

El público objetivo para este estudio lo conforman 382 personas a las cuales se les preguntó sobre el nivel de saturación del mercado de cuero, quienes respondieron tomando en cuenta su experiencia en cuanto a sus marcas favoritas, disponiblidad de los productos, variedad de modelos y precios, entre otros. Por eso, la mitad de los interrogados no están seguros si el mercado de artículos de cuero esté saturado. Para la tercera parte de los encuestados, el mercado no está saturado, dando la pauta de que la marca Legere pueda introducirse a este mercado con más facilidad. Solo la décima parte de los encuestados lo considera asi. 
Considerando que Ecuador tiene un alto reconocimiento mundial en la elaboración de artículos de cuero, ¿usted estaría dispuesto a comprar artículos de este país?

\begin{tabular}{|c|c|c|}
\hline \multicolumn{3}{|c|}{ TABLA DE FRECUENCIA } \\
\hline DETALLE & DATOS & PORCENTAJE \\
\hline $\mathrm{Si}$ & 313 & $81.82 \%$ \\
\hline $\mathrm{No}$ & 69 & $18.18 \%$ \\
\hline TOTAL & $\mathbf{3 8 2}$ & $\mathbf{1 0 0 \%}$ \\
\hline
\end{tabular}

Tabla $N^{\circ}$ 8.- Predisposición para adquirir productos de cuero

Esta interrogante es clave dentro del proyecto ya que indica la predisposición que existe en la población para adquirir productos de cuero. Las cifras que se registran en la parte superior reflejan que el $82 \%$ de las personas encuestadas están predispuestas a adquirir los productos de cuero frente a un $18 \%$ que han manifestado lo contrario.

¿Por cuál medio le gustaría conocer información de una marca ecuatoriana de artículos de cuero?

\begin{tabular}{|c|c|c|}
\hline \multicolumn{3}{|c|}{ TABLA DE FRECUENCIA } \\
\hline DETALLE & DATOS & PORCENTAJE \\
\hline Instagram & 162 & $42.42 \%$ \\
\hline Amazon & 81 & $21.21 \%$ \\
\hline Facebook & 58 & $15.15 \%$ \\
\hline Mailing & 46 & $12.12 \%$ \\
\hline Web & 23 & $6.06 \%$ \\
\hline Otras & 12 & $3.03 \%$ \\
\hline TOTAL & $\mathbf{3 8 2}$ & $\mathbf{1 0 0 \%}$ \\
\hline
\end{tabular}

Tabla $N^{\circ}$ 10.- Medio por el cual gustaría recibir información de la marca

Los medios para difundir la información de la marca son diversos, en esta pregunta se han considerado los más relevantes para así determinar cuál de éstos tiene mayor alcance dentro del público considerado para este estudio. De acuerdo a los encuestados, la red social Instagram es las 
más opcionada con dos cuartas partes de aceptación de la muestra. En segundo lugar está Amazon (compras online) con la quinta parte. De ahí redes como Facebook, mailing y otras portales web se han ubicado con valores alrededor de la décima parte de la muestra.

\section{¿Por cuál medio le gustaría adquirir nuestros productos?}

\begin{tabular}{|c|c|c|}
\hline \multicolumn{3}{|c|}{ TABLA DE FRECUENCIA } \\
\hline DETALLE & DATOS & PORCENTAJE \\
\hline Centro comercial & 150 & $39.39 \%$ \\
\hline Internet & 127 & $33.33 \%$ \\
\hline Tienda especializada & 104 & $27.27 \%$ \\
\hline TOTAL & $\mathbf{3 8 2}$ & $\mathbf{1 0 0 \%}$ \\
\hline
\end{tabular}

\section{Tabla $N^{\circ}$ 11.- Por qué medio le gustaría adquirir los productos Legere}

Finalmente la última interrogante de este proceso de encuesta se direccionó hacia el medio por el cual a los clientes les gustaría adquirir sus productos. Las dos cuartas partes de la muestra prefieren conseguir los produtos de cuero en centros comerciales, tal cual se evidenció en la pregunta 6. Este dato permite centrar la ubicación de las tiendas Legere de manera estratégica en los centros comerciales de la localidad, ya que debido a la concurrencia masiva de personas las ventas de los productos son a gran escala. No por eso deberá desatenderse otros canales, como las ventas online, ya que la tercera parte de los encuestados afirmaron que prefieren ese medio para sus compras. En similar proporción se destacan las tiendas especializadas.

\section{Entrevistas}

Se condujo una entrevista a la propietaria de Legere para conocer la situación actual de la marca en Ecuador, así como sus aspiraciones en el mercado internacional. De acuerdo a la empresaria, al contar con una trayectoria de 3 años, estar posicionada en Guayaquil y poseer un alto estándar de calidad decidió buscar la ampliación en el negocio hacia el exterior. 
Se escogió España por la cercanía, indica la propietaria, y por contar con un contacto que será promotor de la marca en ese país. Se enfoca a un segmento de mercado de consumidores de 30 años en adelante, de estrato socioeconómico medio alto, geográficamente ubicadas en la ciudades principales en Barcelona y Madrid.

Así, su objetivo es conseguir con las ventas internacionales un incremento de las ventas totales del negocio.

\section{Discusión.}

Los productos de Legere son de gran acogida y de acuerdo a las encuestas realizadas, se conoce con mayor exactitud el mercado español. Por ello se realizarán las planificaciones correspondientes para proceder con el suministro de los productos de cuero vacuno al mercado español.

En el desarrollo del presente proyecto es importante mencionar que aunque es un riesgo económico desarrollar una propuesta de internacionalización de los productos, por los costos que conlleva, luego de esta investigación, determinamos que los riesgos se reducen de manera significativa.

Por otro lado, la propuesta es viable gracias a la apertura internacional que brindan los acuerdos comerciales entre Ecuador y la Unión Europea, vigentes a partir de este año. El acuerdo comercial multipartes representa el acceso a un mercado de 16 millones de habitantes de varios productos ecuatorianos con arancel cero, todo esto tras un proceso de desgravamen que se cumplirá de acuerdo a distintos cronogramas. Entre ellos están los artículos manufacturados con cuero 
(maletines, bolsos, maletas y similares, código 42021190), cuyo el arancel aduanero sobre esa mercancía previo a la firma era de $20 \%$.

\section{Conclusiones.}

El mercado de artículos de cuero es sumamente competitivo. El material sobresale a nivel internacional resaltando su importancia tanto para el sector manufacturero como para la economía del país.

Es un mercado de diseño y moda, determinado principalmente por la calidad de material y el diseño. Por tanto, hay que considerar los costos de innovación y diseño en la planificación de las ventas, del marketing y publicidad, conclusión que tiene su base en las respuestas obtenidas en las encuestas, las que señalan que el precio, elegancia, calidad, diseño y moda, ocupan los primeros lugares en el criterio de adquisición de artículos de cuero de los Barceloneses, así: (3.7\%, 3.4\%, $2.9 \%$ y $2.9 \%$ sobre un porcentaje total de 4$)$.

Se ha identificado a España como un mercado atractivo para la comercialización de los artículos de cuero, especialmente en Barcelona, ya que entre el $33.33 \%$ y $51.52 \%$ de los encuestados, consideran que este mercado, no se encuentra saturado.

Además, se logró identificar en base a las encuestas que los barceloneses tienen preferencia para adquirir sus productos a través de centros comerciales, en un $33.39 \%$ y por internet $33.33 \%$, lo que guarda consistencia con los medios de información por los cuales tienen preferencia para obtener información digital, que sitúa a Instagram como la red social más opcionada con dos cuartas partes de aceptación en la muestra, así se encuentra en primer lugar con un 42.42\%, Amazon ( compras on line) 21.21\%, Facebook, ubicada en tercer lugaren con el 15.15\%, se halló que los 
espacios óptimos para la distribución y venta de los artículos son los centros comerciales, sin desatender los canales online, con presencia y difusión en redes sociales y ventas en línea. El precio de los productos de Legere será razonable, siendo altamente competitivo para su introducción y comercialización masiva.

Finalmente, el hecho de que varios productos ecuatorianos, entre ellos los artículos manufacturados de cuero, puedan ingresar en los próximos años a la Unión Europea sin aranceles, facilitará la exportación al reducir los costos. El mercado es atractivo pues, al reducirse esa tarifa, la ganancia es mayor. Además, permite planificar estrategias a largo plazo porque los aranceles serán nulos indefinidamente.

\section{Bibliografía.}

Águeda, E., \& Mondéjar, J. (2013). Fundamentos de Marketing. Madrid: ESIC.

Arciniega, M. (2015). Implementación de estrategias para la exportación de calzado para dama producido por pymes hacia el mercado venezolano. Quito: Universidad Tecnológica Equinoccial.

Avila, C. (2011). Proyecto de Factibilidad de la Exportación de Carteras de cuero al Mercado Alemán, periodo 2011-2020. Quito: Universidad Tecnológica Equinoccial.

Braid, P. (2012). Cuero, Pieles y Calzado. Madrid: Industria textiles y de la confección.

Legere. (2015). Legere, accesorios en cuero y textil. Retrieved 2016 йил octubre from http://www.legere.com.ec 